\title{
SYSTEM OF QUASILINEAR EQUATIONS OF REACTION-DIFFUSION TASKS OF KOLMOGOROV-FISHER TYPE BIOLOGICAL POPULATION TASK IN TWO-DIMENSIONAL CASE
}

\author{
D.K.Muhamediyeva ${ }^{1}$ \\ ${ }^{1}$ Junior researcher of the Centre for development of software products and hardware-software complexes, Tashkent, \\ Uzbekistan
}

\begin{abstract}
In this paper considered parabolic system of two quasilinear reaction-diffusion equations of Kolmogorov-Fisher type of biological population task relation for the two-dimensional case and localization of the wave solutions of the reaction - diffusion systems with double nonlinearity. Cross-diffusion means that spatial move of one object, described by one of the variables is due to the diffusion of another object, described by another variable.Considered spatial analogue of the Volterra-Lotka competition system with nonlinear power dependence of the diffusion coefficient from the population density.
\end{abstract}

Keywords: Population, system, differential equation, cross-diffusion, double nonlinearity, wave solution.

\section{INTRODUCTION}

Let's consider following system of two equations in partial derivatives in two-dimensional case:

$$
\begin{aligned}
& \frac{\partial u_{1}}{\partial t}=f\left(u_{1}, u_{2}\right)+D_{11} \frac{\partial^{2} u_{1}}{\partial x_{1}^{2}}+D_{12} \frac{\partial^{2} u_{1}}{\partial x_{2}^{2}}+h_{11} \frac{\partial}{\partial x_{1}}\left(Q_{1}\left(u_{1}, u_{2}\right) \frac{\partial u_{2}}{\partial x_{1}}\right)+h_{12} \frac{\partial}{\partial x_{2}}\left(Q_{1}\left(u_{1}, u_{2}\right) \frac{\partial u_{2}}{\partial x_{2}}\right) \\
& \frac{\partial u_{2}}{\partial t}=g\left(u_{1}, u_{2}\right)+D_{21} \frac{\partial^{2} u_{2}}{\partial x_{1}^{2}}+D_{22} \frac{\partial^{2} u_{2}}{\partial x_{2}^{2}}+h_{21} \frac{\partial}{\partial x_{1}}\left(Q_{2}\left(u_{1}, u_{2}\right) \frac{\partial u_{1}}{\partial x_{1}}\right)+h_{22} \frac{\partial}{\partial x_{2}}\left(Q_{2}\left(u_{1}, u_{2}\right) \frac{\partial u_{1}}{\partial x_{2}}\right) .
\end{aligned}
$$

At $h_{11}=h_{12}=h_{21}=h_{22}=0$ the mathematical model (1) is a system type reaction-diffusion with diffusion coefficient $D_{11} \geq 0, D_{12} \geq 0, D_{21} \geq 0, D_{22} \geq 0 \quad$ (at least $\quad$ one $\left.D_{i j} \neq 0\right)$. In the case when at least one of the coefficients (mark can be any), the system (1) is a cross-diffusion. To the linear cross-diffusion corresponds $Q_{i j}(u, v)=$ const for $i, j=1,2 \quad \mathrm{i}=1,2 ; \quad$ to $\quad$ the linear cross-diffusion$Q_{i j}(u, v) \neq$ const at least one of $\mathrm{i}$ and $\mathrm{j}$.

Cross-diffusion means that spatial move one object, described one of the variables is due to the diffusion of another object, described by another variable.At the population level simplest example is a parasite (the first object, located within the "host" (the second object) moves through the diffusion of the owner). The term "selfdiffusion" (diffuse, direct diffusion, ordinary diffusion) moves individuals at the expense of the diffusion flow from areas of high concentration, particularly in the area of low concentration. The term "cross-diffusion" means moving/thread one species/ substances due to the presence of the gradient other individuals/ substances. B The value of a cross-diffusion coefficient can be positive, negative or equal to zero. The positive coefficient of cross-diffuse indicates that the movement of individuals takes place in the direction of low concentrations of other species occurs in the direction of the high concentration of other types of individuals/ substances. In the nature system with crossdiffusion quite common and play a significant role especially in biophysical and biomedical systems.

Equation (1) is a generalization of the simple diffusion model for the logistic model of population growth [1-16] of Malthus type $\left(f_{1}\left(u_{1}, u_{2}\right)=u_{1}, f_{1}\left(u_{1}, u_{2}\right)=u_{2}\right.$, $\left.f_{2}\left(u_{1}, u_{2}\right)=u_{1}, \quad f_{2}\left(u_{1}, u_{2}\right)=u_{2}\right)$, Ferhulst type ( $f_{1}\left(u_{1}, u_{2}\right)=u_{1}\left(1-u_{2}\right), f_{1}\left(u_{1}, u_{2}\right)=u_{2}\left(1-u_{1}\right)$, $\left.f_{2}\left(u_{1}, u_{2}\right)=u_{1}\left(1-u_{2}\right), f_{2}\left(u_{1}, u_{2}\right)=u_{2}\left(1-u_{1}\right)\right)$, and Allee type $\quad\left(f_{1}\left(u_{1}, u_{2}\right)=u_{1}\left(1-u_{2}{ }^{\beta_{1}}\right)\right.$, $f_{1}\left(u_{1}, u_{2}\right)=u_{2}\left(1-u_{1}^{\beta_{2}}\right), \quad f_{2}\left(u_{1}, u_{2}\right)=u_{1}\left(1-u_{2}^{\beta_{1}}\right)$, $\left.f_{2}\left(u_{1}, u_{2}\right)=u_{2}\left(1-u_{1}^{\beta_{2}}\right), \beta_{1}>1, \beta_{2}>1\right)$ for the case 
of double nonlinear diffusion. In case, when $\beta_{1} \geq 1, \beta_{2} \geq 1$, it can be regarded as the equation of nonlinear filtration, thermal conductivity at simultaneous influence of the source and the absorption capacity of which is equalrespectively to $u_{1},-u_{2}^{\beta_{1}}, u_{2},-u_{1}^{\beta_{2}}$.

Let's consider the spatial analogue of Volterra-Lotka competition system with nonlinear power dependence of the diffusion coefficient from population density. In the case of the simplest Volterra's competitive interactions between populations can be constructed numerically, and in some cases analytically heterogeneous in space solutions[19].

\section{LOCALIZATION OF WAVE SOLUTIONS OF REACTION - DIFFUSION SYSTEMS WITH DOUBLE NONLINEARITY}

Let's consider in the domain $\mathrm{Q}=\left\{(\mathrm{t}, \mathrm{x}): 0<\mathrm{t}<\infty, \mathrm{x} \in \mathrm{R}^{2}\right\}$ parabolic system of two quasilinear equations of reactiondiffusion of Kolmogorov-Fisher typebiological population task

$$
\left\{\begin{array}{c}
\frac{\partial u_{1}}{\partial t}=\frac{\partial}{\partial x_{1}}\left(D_{11} u_{2}^{m_{1}-1}\left|\frac{\partial u_{1}}{\partial x_{1}}\right|^{p-2} \frac{\partial u_{1}}{\partial x_{1}}\right)+\frac{\partial}{\partial x_{2}}\left(D_{12} u_{2}^{m_{1}-1}\left|\frac{\partial u_{1}}{\partial x_{2}}\right|^{p-2} \frac{\partial u_{1}}{\partial x_{2}}\right)+l_{1}(t) \frac{\partial u_{1}}{\partial x_{1}}+l_{2}(t) \frac{\partial u_{1}}{\partial x_{2}}+k_{1}(t) u_{1}\left(1-u_{2}^{\beta_{1}}\right), \\
\left.\frac{\partial u_{2}}{\partial t}=\frac{\partial}{\partial x_{1}}\left(D_{21} u_{1}^{m_{2}-1}\left|\frac{\partial u_{2}}{\partial x_{1}}\right|^{p-2} \frac{\partial u_{2}}{\partial x_{1}}\right)+\left.\frac{\partial}{\partial x_{2}}\left|D_{2} u_{1}^{m_{2}-1}\right| \frac{\partial u_{2}}{\partial x_{2}}\right|^{p-2} \frac{\partial u_{2}}{\partial x_{2}}\right)+l_{1}(t) \frac{\partial u_{2}}{\partial x_{1}}+l_{2}(t) \frac{\partial u_{2}}{\partial x_{2}}+k_{2}(t) u_{2}\left(1-u_{1}^{\beta_{2}}\right), \\
\left.u_{1}\right|_{t=0}=u_{10}(x),\left.u_{2}\right|_{t=0}=u_{20}(x),
\end{array}\right.
$$

which describes the process of biological populations in nonlinear two-component environment, it's diffusion coefficient is equal $D_{11} u_{2}^{m_{1}-1}\left|\frac{\partial u_{1}}{\partial x_{1}}\right|^{p-2}, D_{12} u_{2}^{m_{1}-1}\left|\frac{\partial u_{1}}{\partial x_{2}}\right|^{p-2}$, $D_{21} u_{1}^{m_{2}-1}\left|\frac{\partial u_{2}}{\partial x_{1}}\right|^{p-2}, \quad D_{22} u_{1}^{m_{2}-1}\left|\frac{\partial u_{2}}{\partial x_{2}}\right|^{p-2} \quad$ andconvective transfer with speed $l_{i}(t)$, where $m_{1}, m_{2}, p, \beta_{1}, \beta_{2}$ positive real numbers, $u_{1}=u_{1}\left(t, x_{1}, x_{2}\right) \geq 0$, $u_{2}=u_{2}\left(t, x_{1}, x_{2}\right) \geq 0$-search solutions.

The Cauchy problem and boundary problems for the system (1) in one-dimensional and multidimensional cases investigated by many authors [15-21]

The aim of this work is the investigation of qualitative properties of solutions of the task (2) on the basis of selfsimilar analysis and numerical solutions by using the methods of modern computer technologies, research of methods of linearization to the convergence of iterative process with subsequent visualization. Obtained the estimates of solutions and emerging in this case free boundary, that gives the chance to choose the appropriate initial approximation [15] for each value of the numeric parameters.

It is known that the nonlinear wave equations have solutions in the form of diffusion waves. Under the wave is understood self-similar solution of the equation (2)

$$
\begin{gathered}
u(t, x)=f(\xi), \xi=c t \pm x \\
u(t, x)=u\left(t, x_{1}, x_{2}\right), \quad x=\sqrt{\left(x_{1}\right)^{2}+\left(x_{2}\right)^{2}}
\end{gathered}
$$

Where constant c- is a wave speed

Let's build self-similar system of equations (2) - more simple for research system of equations.

Self-similar system of equations will construct by the method of nonlinear splitting [15].

Replacement in (2)

$$
\begin{aligned}
u_{1}\left(t, x_{1}, x_{2}\right) & =e^{-\int_{0}^{t} k_{1}(\zeta) d \zeta} v_{1}\left(\tau(t), \eta_{1}, \eta_{2}\right), \\
\eta_{1}=x_{1}-\int_{0}^{t} l_{1}(\zeta) d \zeta, & \\
u_{2}\left(t, x_{1}, x_{2}\right) & =e^{-\int_{0}^{t} k_{2}(\zeta) d \zeta} v_{2}\left(\tau(t), \eta_{1}, \eta_{2}\right), \\
\eta_{2} & =x_{2}-\int_{0}^{t} l_{2}(\zeta) d \zeta,
\end{aligned}
$$


Lead (2) to the form:

$$
\left\{\begin{array}{c}
\frac{\partial v_{1}}{\partial \tau}=\frac{\partial}{\partial \eta_{1}}\left(D_{11} v_{2}^{m_{1}-1}\left|\frac{\partial v_{1}}{\partial \eta_{1}}\right|^{p-2} \frac{\partial v_{1}}{\partial \eta_{1}}\right)+\frac{\partial}{\partial \eta_{2}}\left(D_{12} v_{2}^{m_{1}-1}\left|\frac{\partial v_{1}}{\partial \eta_{2}}\right|^{p-2} \frac{\partial v_{1}}{\partial \eta_{2}}\right)-k_{1}(t) e^{\left[(2-p) k_{1}+\left(\beta_{1}-m_{1}+1\right) k_{2}\right] t} v_{1} v_{2}^{\beta_{1}} \\
\frac{\partial v_{2}}{\partial \tau}=\frac{\partial}{\partial \eta_{1}}\left(D_{21} v_{1}^{m_{2}-1}\left|\frac{\partial v_{2}}{\partial \eta_{1}}\right|^{p-2} \frac{\partial v_{2}}{\partial \eta_{1}}\right)+\frac{\partial}{\partial \eta_{2}}\left(D_{22} v_{1}^{m_{2}-1}\left|\frac{\partial v_{2}}{\partial \eta_{2}}\right|^{p-2} \frac{\partial v_{2}}{\partial \eta_{2}}\right)-k_{2}(t) e^{\left[\left(\beta_{2}-m_{2}+1\right) k_{1}+(2-p) k_{2}\right] t} v_{1}^{\beta_{2}} v_{2}, \\
\left.v_{1}\right|_{t=0}=v_{10}\left(\eta_{1}, \eta_{2}\right),\left.v_{2}\right|_{t=0}=v_{20}\left(\eta_{1}, \eta_{2}\right) .
\end{array}\right.
$$

If $k_{1}\left(p-\left(m_{1}+1\right)\right)=k_{2}\left(p-\left(m_{2}+1\right)\right), \quad$ then $\quad$ by choosing

$$
\tau(t)=\frac{e^{\left[\left(m_{1}-1\right) k_{2}+(p-2) k_{1}\right] t}}{\left(m_{1}-1\right) k_{2}+(p-2) k_{1}}=\frac{e^{\left[\left(m_{2}-1\right) k_{1}+(p-2) k_{2}\right] t}}{\left(m_{2}-1\right) k_{1}+(p-2) k_{2}}
$$
,we get the following system of equations:

$$
\left\{\begin{array}{l}
\frac{\partial v_{1}}{\partial \tau}=\frac{\partial}{\partial \eta_{1}}\left(D_{11} v_{2}^{m_{1}-1}\left|\frac{\partial v_{1}}{\partial \eta_{1}}\right|^{p-2} \frac{\partial v_{1}}{\partial \eta_{1}}\right)+\frac{\partial}{\partial \eta_{2}}\left(D_{12} v_{2}^{m_{1}-1}\left|\frac{\partial v_{1}}{\partial \eta_{2}}\right|^{p-2} \frac{\partial v_{1}}{\partial \eta_{2}}\right)-a_{1}(t) \tau^{b_{1}} v_{1} v_{2}^{\beta_{1}}, \\
\frac{\partial v_{2}}{\partial \tau}=\frac{\partial}{\partial \eta_{1}}\left(D_{21} v_{1}^{m_{2}-1}\left|\frac{\partial v_{2}}{\partial \eta_{1}}\right|^{p-2} \frac{\partial v_{2}}{\partial \eta_{1}}\right)+\frac{\partial}{\partial \eta_{2}}\left(D_{22} v_{1}^{m_{2}-1}\left|\frac{\partial v_{2}}{\partial \eta_{2}}\right|^{p-2} \frac{\partial v_{2}}{\partial \eta_{2}}\right)-a_{2}(t) \tau^{b_{2}} v_{1}^{\beta_{2}} v_{2},
\end{array}\right.
$$

where $a_{1}=k_{1}\left((p-2) k_{1}+\left(m_{1}-1\right) k_{2}\right)^{b_{1}}, b_{1}=\frac{(2-p) k_{1}+\left(\beta_{1}-m_{1}+1\right) k_{2}}{(p-2) k_{1}+\left(m_{1}-1\right) k_{2}}$,

$a_{2}=k_{2}\left(\left(m_{2}-1\right) k_{1}+(p-2) k_{2}\right)^{b_{2}}, b_{2}=\frac{\left(\beta_{2}-m_{2}+1\right) k_{1}+(2-p) k_{2}}{\left(m_{2}-1\right) k_{1}+(p-2) k_{2}}$.

If $b_{i}=0$, and $a_{i}(t)=$ const $, i=1,2$, then the system has the form:

$$
\left\{\begin{array}{l}
\frac{\partial v_{1}}{\partial \tau}=\frac{\partial}{\partial \eta_{1}}\left(D_{11} v_{2}^{m_{1}-1}\left|\frac{\partial v_{1}}{\partial \eta_{1}}\right|^{p-2} \frac{\partial v_{1}}{\partial \eta_{1}}\right)+\frac{\partial}{\partial \eta_{2}}\left(D_{12} v_{2}^{m_{1}-1}\left|\frac{\partial v_{1}}{\partial \eta_{2}}\right|^{p-2} \frac{\partial v_{1}}{\partial \eta_{2}}\right)-a_{1} v_{1} v_{2}^{\beta_{1}} \\
\frac{\partial v_{2}}{\partial \tau}=\frac{\partial}{\partial \eta_{1}}\left(D_{21} v_{1}^{m_{2}-1}\left|\frac{\partial v_{2}}{\partial \eta_{1}}\right|^{p-2} \frac{\partial v_{2}}{\partial \eta_{1}}\right)+\frac{\partial}{\partial \eta_{2}}\left(D_{22} v_{1}^{m_{2}-1}\left|\frac{\partial v_{2}}{\partial \eta_{2}}\right|^{p-2} \frac{\partial v_{2}}{\partial \eta_{2}}\right)-a_{2} v_{1}^{\beta_{2}} v_{2}
\end{array}\right.
$$

The Cauchy problem for system (4) in the case when $b_{1}=b_{2}=0$ studied in $[16,19]$ and prove the existence of wave global solutions and blow-up solutions.

Below we will describe one way of obtaining self-similar system for the system of equations (4). It consists in the following. Firstly we find first the solution of a system of ordinary differential equations

$$
\left\{\begin{array}{l}
\frac{d \overline{\nu_{1}}}{d \tau}=-a_{1} \bar{\nu}_{1}{\overline{\nu_{2}}}^{\beta_{1}}, \\
\frac{d \overline{\nu_{2}}}{d \tau}=-a_{2}{\overline{v_{1}}}^{\beta_{2}}{\overline{\nu_{2}}},
\end{array}\right.
$$

In the form

$\overline{v_{1}}(\tau)=c_{1}\left(\tau+T_{0}\right)^{-\gamma_{1}}, \bar{v}_{2}(\tau)=c_{2}\left(\tau+T_{0}\right)^{-\gamma_{2}}, T_{0}>0$

Where 
$c_{1}=1, \gamma_{1}=\frac{1}{\beta_{2}}, c_{2}=1, \gamma_{2}=\frac{1}{\beta_{1}}$.

And then the solution of system (3)-(4) is sought in the form

$$
\begin{aligned}
& v_{1}\left(t, \eta_{1}, \eta_{2}\right)=\bar{v}_{1}(t) w_{1}\left(\tau, \eta_{1}, \eta_{2}\right), \\
& v_{2}\left(t, \eta_{1}, \eta_{2}\right)=\bar{v}_{2}(t) w_{2}\left(\tau, \eta_{1}, \eta_{2}\right),
\end{aligned}
$$

$\tau_{1}(\tau)=\int_{0}^{\tau} \bar{v}_{1}^{(p-2)}(t) \bar{v}_{2}^{\left(m_{1}-1\right)}(t) d t= \begin{cases}\frac{1}{1-\left[\gamma_{1}(p-2)+\gamma_{2}\left(m_{1}-1\right)\right]}(T+\tau)^{1-\left[\gamma_{1}(p-2)+\gamma_{2}\left(m_{1}-1\right)\right]}, \text { if } 1-\left[\gamma_{1}(p-2)+\gamma_{2}\left(m_{1}-1\right)\right] \neq 0, \\ \ln (T+\tau), & \text { if } 1-\left[\gamma_{1}(p-2)+\gamma_{2}\left(m_{1}-1\right)\right]=0, \\ (T+\tau), & \text { if } p=2 u m_{1}=1,\end{cases}$

if $\gamma_{1}(p-2)+\gamma_{2}\left(m_{1}-1\right)=\gamma_{2}(p-2)+\gamma_{1}\left(m_{2}-1\right) . \quad$ Then for $w_{i}(\tau, x), i=1,2$ we get the following system of equations

$$
\left\{\begin{array}{l}
\frac{\partial w_{1}}{\partial \tau}=\frac{\partial}{\partial \eta_{1}}\left(D_{11} w_{2}^{m_{1}-1}\left|\frac{\partial w_{1}}{\partial \eta_{1}}\right|^{p-2} \frac{\partial w_{1}}{\partial \eta_{1}}\right)+\frac{\partial}{\partial \eta_{2}}\left(D_{12} w_{2}^{m_{1}-1}\left|\frac{\partial w_{1}}{\partial \eta_{2}}\right|^{p-2} \frac{\partial w_{1}}{\partial \eta_{2}}\right)+\psi_{1}\left(w_{1} w_{2}^{\beta_{1}}-w_{1}\right) \\
\frac{\partial w_{2}}{\partial \tau}=\frac{\partial}{\partial \eta_{1}}\left(D_{21} w_{1}^{m_{2}-1}\left|\frac{\partial w_{2}}{\partial \eta_{1}}\right|^{p-2} \frac{\partial w_{2}}{\partial \eta_{1}}\right)+\frac{\partial}{\partial \eta_{2}}\left(D_{22} w_{1}^{m_{2}-1}\left|\frac{\partial w_{2}}{\partial \eta_{2}}\right|^{p-2} \frac{\partial w_{2}}{\partial \eta_{2}}\right)+\psi_{2}\left(w_{2} w_{1}^{\beta_{2}}-w_{2}\right)
\end{array}\right.
$$

Where

$$
\begin{aligned}
& \psi_{1}= \begin{cases}\frac{1}{\left(1-\left[\gamma_{1}(p-2)+\gamma_{2}\left(m_{1}-1\right)\right]\right) \tau}, & \text { if } 1-\left[\gamma_{1}(p-2)+\gamma_{2}\left(m_{1}-1\right)>0,\right. \\
\left.\gamma_{1} c_{1}^{-\left(1-\gamma_{1}(p-2)+\gamma_{2}\left(m_{1}-1\right)\right]}\right), & \text { if } 1-\left[\gamma_{1}(p-2)+\gamma_{2}\left(m_{1}-1\right)=0,\right.\end{cases} \\
& \psi_{2}= \begin{cases}\frac{1}{\left(1-\left[\gamma_{2}(p-2)+\gamma_{1}\left(m_{2}-1\right)\right]\right) \tau}, & \text { if } 1-\left[\gamma_{2}(p-2)+\gamma_{1}\left(m_{2}-1\right)\right]>0, \\
\gamma_{2} c_{1}^{-\left(1-\left[\gamma_{2}(p-2)+\gamma_{1}\left(m_{2}-1\right)\right]\right)}, & \text { if } 1-\left[\gamma_{2}(p-2)+\gamma_{1}\left(m_{2}-1\right)\right]=0 .\end{cases}
\end{aligned}
$$

Therefore, the solution of system (1) with condition (5) tends to the solution of the system (8). 
If $1-\left[\gamma_{1}(p-2)+\gamma_{2}\left(m_{1}-1\right)=0\right.$, wave solution of system $(6)$ has the form

$$
w_{i}\left(\tau(t), \eta_{1}, \eta_{2}\right)=y_{i}(\xi), \xi=c \tau \pm \eta, \quad \eta=\sqrt{\left(\eta_{1}\right)^{2}+\left(\eta_{2}\right)^{2}} i=1,2
$$

where c- wave velocity, and taking into account that the equation for $w_{i}\left(\tau, \eta_{1}, \eta_{2}\right)$ without the younger members always has a self-similar solution if $1-\left[\gamma_{1}(p-2)+\gamma_{2}\left(m_{1}-1\right) \neq 0\right.$ we get the system

$$
\left\{\begin{array}{l}
\frac{d}{d \xi}\left(y_{2}^{m_{1}-1}\left|\frac{d y_{1}}{d \xi}\right|^{p-2} \frac{d y_{1}}{d \xi}\right)+c \frac{d y_{1}}{d \xi}+\psi_{1}\left(y_{1}-y_{1} y_{2}^{\beta_{1}}\right)=0, \\
\frac{d}{d \xi}\left(y_{1}^{m_{2}-1}\left|\frac{d y_{2}}{d \xi}\right|^{p-2} \frac{d y_{2}}{d \xi}\right)+c \frac{d y_{2}}{d \xi}+\psi_{2}\left(y_{2}-y_{2} y_{1}^{\beta_{2}}\right)=0 .
\end{array}\right.
$$

After integration (8) we get the system of nonlinear differential equations of the first order

$$
\left\{\begin{array}{l}
y_{2}^{m_{1}-1}\left|\frac{d y_{1}}{d \xi}\right|^{p-2} \frac{d y_{1}}{d \xi}+c y_{1}=0 \\
y_{1}^{m_{2}-1}\left|\frac{d y_{2}}{d \xi}\right|^{p-2} \frac{d y_{2}}{d \xi}+c y_{2}=0
\end{array}\right.
$$

The system (9) has an approximate solution in the form

$$
\bar{y}_{1}=A(a-\xi)^{\gamma_{1}}, \bar{y}_{2}=B(a-\xi)^{\gamma_{2}}
$$

Where

$$
\begin{aligned}
& \gamma_{1}=\frac{(p-1)\left(p-\left(m_{1}+1\right)\right)}{(p-2)^{2}-\left(m_{1}-1\right)\left(m_{2}-1\right)}, \\
& \gamma_{2}=\frac{(p-1)\left(p-\left(m_{2}+1\right)\right)}{(p-2)^{2}-\left(m_{1}-1\right)\left(m_{2}-1\right)} .
\end{aligned}
$$

and the coefficients $\mathrm{A}$ and $\mathrm{B}$ are determined from the solution of a system of nonlinear algebraic equations $\left(\gamma_{1}\right)^{p-1} A^{p-1} B^{m_{1}-1}=c$,

$$
\left(\gamma_{2}\right)^{p-1} A^{m_{2}-1} B^{p-1}=c
$$

Then by taking into account expressions

$$
u_{1}\left(t, x_{1}, x_{2}\right)=e^{-\int_{0}^{t} k_{1}(\zeta) d \zeta} v_{1}\left(\tau(t), \eta_{1}, \eta_{2}\right)
$$

$$
u_{2}\left(t, x_{1}, x_{2}\right)=e^{-\int_{0}^{t} k_{2}(\zeta) d \zeta} v_{2}\left(\tau(t), \eta_{1}, \eta_{2}\right)
$$

We have

$$
\begin{gathered}
u_{1}\left(t, x_{1}, x_{2}\right)=A e^{-\int_{0}^{t} k_{1}(\zeta) d \zeta}(c \tau(t)-\xi)_{+}^{\gamma_{1}}, \\
u_{2}\left(t, x_{1}, x_{2}\right)=B e^{-\int_{0}^{t} k_{2}(\zeta) d \zeta}(c \tau(t)-\xi)_{+}^{\gamma_{2}}, c>0 .
\end{gathered}
$$

Due to the fact that

$$
\left[b \tau(t)-\int_{0}^{t} l_{i}(\eta) d \eta-x_{i}\right]=0
$$

If

$$
x_{i} \geq\left[b \tau(t)-\int_{0}^{t} l_{i}(\eta) d \eta-x_{i}\right]<0, \forall t>0,
$$

Then

$$
\begin{aligned}
& u_{1}(t, x) \equiv 0, u_{2}(t, x) \equiv 0 \\
& x_{i} \geq\left[b \tau(t)-\int_{0}^{t} l_{i}(\eta) d \eta-x_{i}\right]<0, \forall t>0, \quad i=1,2
\end{aligned}
$$

Therefore, localization condition of solutions of the system (2) are conditions

$$
\int_{0}^{a} l_{i}(y) d y<0, \tau(t)<\infty \text { for } \forall t>0, i=1,2 .
$$


Condition (10) is the prerequisite for the emergence of a new effect - localizationof wave solutions of (2). If condition (10) unfulfilled, then there is the phenomenon of the finite velocity of propagation of a perturbation, i.e.

$u_{i}(t, x) \equiv 0$ at $|x| \geq b(t), \tau(t)=\int_{0}^{t} e-^{\left(m_{1}+p-3\right) \int_{0}^{\zeta} k_{1}(y) d y} d \zeta$ , and the front of arbitrarily far away, with increasing time, since $\tau(t) \rightarrow \infty$ at $t \rightarrow \infty$.

The study of qualitative properties of the system (2) allowed to perform numerical experiment based on the values included in the system of numeric parameters. For this purpose, as the initial approximation was used asymptotic solutions. To numerical solving the task for the linearization of system (2) has been used linearization methods of Newton and Picard. To build self-similar system of equationsof biological populations used the method of nonlinear splitting $[16,19]$.

\section{NUMERICAL EXPERIMENT}

Let's construct a uniform grid to the numericalsolvingof the task (2)

$$
\omega_{h}=\left\{x_{i}=i h, \quad h>0, i=0,1, \ldots, n, \quad h n=l\right\}
$$

and the temporary grit

$$
\omega_{h_{1}}=\left\{t_{j}=j h_{1}, \quad h_{1}>0, j=0,1, \ldots, n, \quad \tau m=T\right\}
$$

As is known, the main problem for the numerical solving of nonlinear tasks, is suitable choosing initial approximation and the linearization method of the system (2). Let's consider the function:

$$
\begin{gathered}
v_{10}\left(t, x_{1}, x_{2}\right)=v_{1}(t) \cdot(a-\xi)_{+}^{\gamma_{1}}, \\
v_{20}\left(t, x_{1}, x_{2}\right)=v_{2}(t) \cdot(a-\xi)_{+}^{\gamma_{2}},
\end{gathered}
$$

Where $v_{1}(t)=e^{k t} \bar{v}_{1}(t)$ and $v_{2}(t)=e^{k t} \bar{v}_{2}(t)$ defined above functions,

Notation $(a)_{+}$means $(a)_{+}=\max (0, a)$. These functions have the property of finite speed of propagation of perturbations[16,19]. Therefore, in the numerical solving of the task (1) - (2) at $\beta_{1}>\sigma_{1}$ as an initial approximation proposed the function $v_{i 0}\left(t, x_{1}, x_{2}\right), i=1,2$.

Created on input language MathCad program allows you to visually traced the evolution of the process for different values of the parameters and data.

Numerical calculations show that in the case of arbitrary values $\sigma>0, \beta>0$ qualitative properties of solutions do not change. Below listed results of numerical experiments for different values of parameters (Fig.1)

Replace task (2) by implicit difference scheme and receive differential task with the error $O\left(h^{2}+h_{1}\right)$.

\begin{tabular}{|l|r|}
\hline Parameter values & Results of numerical experiment \\
\hline $\begin{array}{l}m_{1}=0.3, m_{2}=0.7, p=2.1 \\
\beta_{1}=5, k_{1}=2\end{array}$ & \\
$\beta_{2}=7, k_{2}=3$ & \\
$e p s=10^{-3}$ & \\
& \\
& \\
& \\
& \\
& \\
\end{tabular}




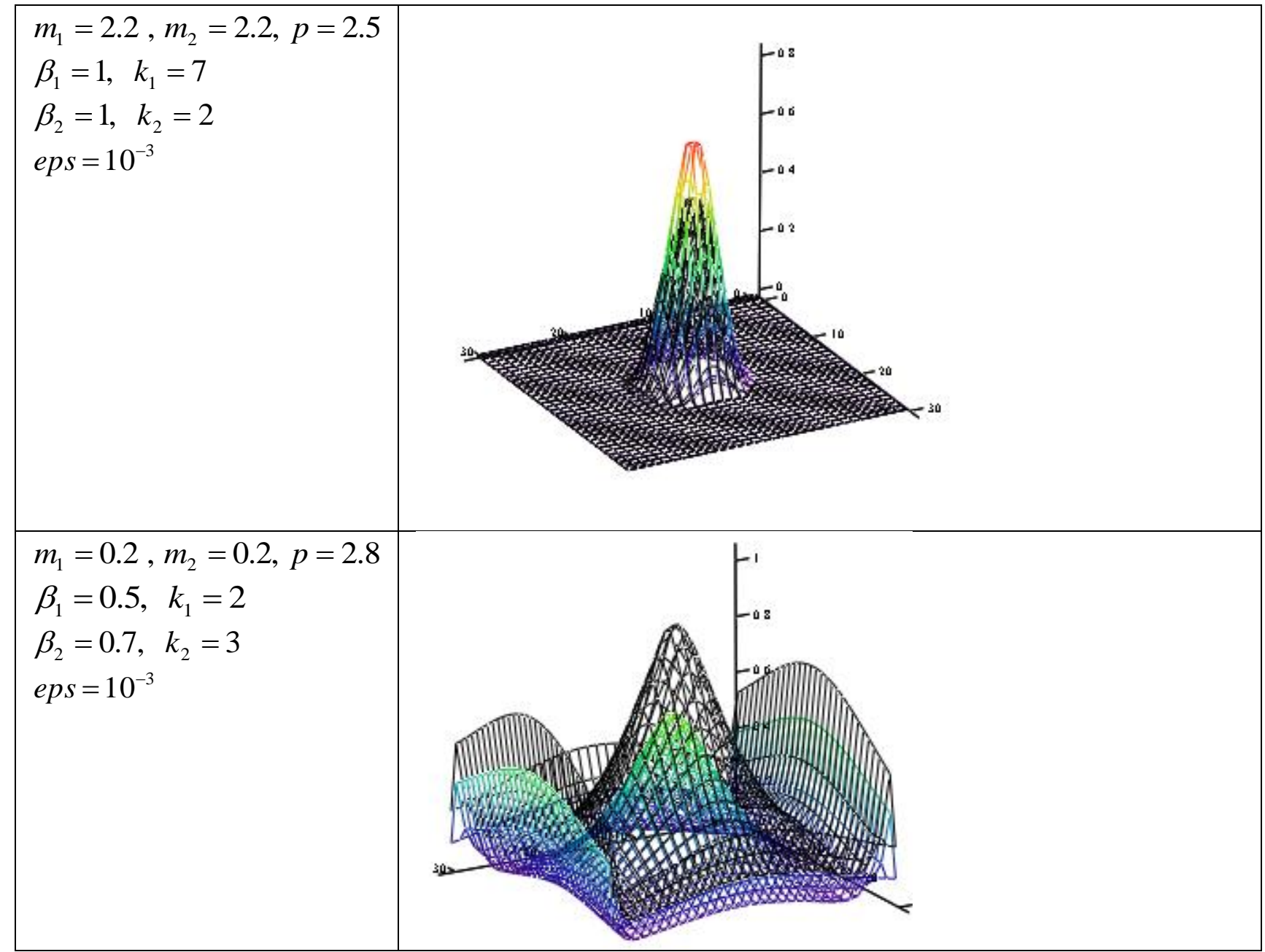

Fig.1 Results of numerical experiment

\section{CONCLUSIONS}

It can be expected that further theoretical and experimental studies of excitable systems with cross-diffusion will make a significant contribution to the study of phenomena of selforganization in all of nonlinear systems from the micro and astrophysical systems to public social systems.

\section{REFERENCES}

[1] Ахромеева ТСи др. Нестационарные структуры и диффузионный хаос (М.: Наука, 1992)

[2] de1-Casti11o-Negrete D, Carreras B A Phys. Plasmas 9118 (2002)

[3] del-Castillo-Negrete D, Сагге B A, Lynch V Physica D 168-169 45 (2002)

[4] Jorne J L Theor. Biol. 55529 (1975)

[5] A1mirantis Y, Papageorgiou S L Theor. Biol. 151 289 (1991)

[6] Иваницкий Г Р, Медвинский А Б, Цыганов М А УФH161 (4) 13 (1991)

[7] Wu Y, Zhao X Physica D 200325 (2005)

[8] Kuznetsov Yu A et a1. L Math. Biol. 32219 (1994)

[9] Кузнецов Ю А и др. "Кросс-диффузионная модель динамики границы леса", в сб. Проблемь экологического мониторинга и моделирования экосистем Т. 26 (СПб.: Гидрометиздат, 1996) с. 213

[10] Burridge R, KnopoffL Bм//. Seismol. Soc. Am. 57 341 (1967)

[11] Cartwright J HE, Hernandez-Garcia E, Piro O Phys. Pev. Lett. 79 527(1997)

[12] Stuart A M /MA L Math. Appl. Med. Biol. 10149 (1993)

[13] Murray J.D. Mathematical Biology. I. An Introduction (Third Edition). - N.Y., Berlin, Heidelberg: Springer Verlag, 2001. - 551 p.,

[14] M. Aripov (1997). «ApproximateSelfsimilarApproachtuSolve Quasilinear Parabolic Equation» Experimentation, Modeling and Computation in Flow Turbulence and Combustion vol. 2. p. 19- 26.

[15] Арипов М. Метод эталонных уравнений для решения нелинейных краевых задач Ташкент, Фан, 1988, 137 p.

[16] Белотелов Н.В., Лобанов А.И.Популяционные модели с нелинейной диффузией. // Математическое моделирование. -М.; 1997, №12, pp. 43-56.

[17] В. Вольтерра. Математическая теория борьбы за существование -М.: Наука, 1976, 288 р. 
[18] Гаузе Г.Ф. О процессах уничтожения одного вида другим в популяциях инфузорий // Зоологический журнал, 1934, т.13, №1.

[19] Aripov M., Muhammadiev J. Asymptotic behaviour of automodel solutions for one system of quasilinear equations of parabolic type. Buletin StiintificUniversitatea din Pitesti, Seria Matematica si Informatica. N 3. 1999. pg. 19-40

[20] Aripov M.M. Muhamediyeva D.K. To the numerical modeling of self-similar solutions of reactiondiffusion system of the one task of biological population of Kolmogorov-Fisher type. International Journal of Engineering and Technology. Vol-02, Iss11, Nov-2013. India. 2013.

[21] Арипов М.М. Мухамедиева Д.К. Подходы к решению одной задачи биологической популяции. Вопросы вычислительной и прикладной математики. -Ташкент. 2013. Вып.129.-pp.22-31. 